\title{
Correlation Of Imaging Technologies: Methodologies.
}

Claudia S. López ${ }^{* 1,2,3}$, Kevin Loftis ${ }^{2}$, Guillaume Thibault ${ }^{2}$, Sunjong Kwon ${ }^{2}$, Erin Stempinski ${ }^{1,2}$, Jessica L. Riesterer ${ }^{1,2}$, Joe W. Gray ${ }^{2}$

${ }^{1}$ Multiscale Microscopy Core, Oregon Health \& Science University (OHSU).

2. OHSU Center for Spatial Systems Biomedicine (OCSSB).

3. Pacific Northwest Center for CryoEM, Portland Oregon USA.

*Corresponding author: lopezcl@ohsu.edu

Correlative technologies have been transforming the way researchers obtain information from their specimens based on the advantages of these different technologies that range between the millimeter and nanometer scales. Attempts to combine multidimensional data are often met with the challenge of overcoming nonideal sample conditions such as reduced fluorescence signal, poor specimen preservation, specimen deformation, or low specimen contrast, prompting the development and use of enhanced sample preparation procedures to overcome such challenges. Correlative light and electron microscopy (CLEM) can be done by means of transmission (TEM) and scanning (SEM) electron imaging (Figure 1), and depending on the instrument of choice could also include 3D volume acquisition (Figure 2) [1,2].

In recent years, we have optimized methods to correlate cell imaging with $2 \mathrm{D}$ SEM and 3D Focused Ion Beam-SEM (FIB-SEM) (Figure 2) [3, 4], to correlate superresolution imaging with SEM [5], to preserve fluorescent signal in methacrylate resins as shown in Figure 1 [6]. Additionally, alongside collaborators, we have developed genetically encoded peptide tags for use in CLEM imaging [7]. More recently, our focus has been to develop methods to correlate micro computed tomography (microCT) and Serial Block face SEM (SBF-SEM) (Rykiel et. al in preparation). In all these cases, sample optimization was necessary in order to achieve maximum sample quality for both imaging platforms. Sequential CLEM has the advantage that the sample is imaged first using any optical method of interest and it is later processed either for 2D or 3D EM (Figure 2) only if the optical imaging was successful. In this workflow the introduction of heavy metals needed for both 3D FIB-SEM or 3D SBF-SEM is done in later steps to ensure that the fluorescent signal is not compromised [4]. In the case of the in-resin fluorescence preservation optimizations we have pursued (Figure 1), we have observed an inconsistent behavior of London Resin White (LRW) indicating batch dependency. It is therefore important to first check the polymerization characteristics of any new batch of LRW resin before its use. In our hands, in-resin preservation methods include polymerizations at $50^{\circ} \mathrm{C}$ or $37^{\circ} \mathrm{C}$ depending on the fluorophore. Polymerization at $37^{\circ} \mathrm{C}$ can be achieved by adjusting the $\mathrm{pH}$ of the LRW utilized closer to neutral with triethanolamine. The addition of a basic solution of sodium carbonate $\mathrm{pH} 9.0$ for en block or methacrylate plastic sections fluorescence imaging recovers, in most cases, the fluoresce properties of genetically encoded green fluorescent proteins (GFP) [6]. Finally, the presence of internal or external fiducial markers is very important for any CLEM workflow. These can be either added to the specimen (gold fiducials, nanopsheres, etc.), can be part of the specimen (features within the samples, shape, etc.) or can be embedded on the sample holder or substrate of choice $[1,4,5,7]$. In all cases, a correlative image software is a key player to bridging the differences in resolution, which enables the integration of molecular and structural information into one common framework. There are several software programs, both open source or licensed, available for such 
correlation and include rigid and nonrigid registrations of the images. Depending on the protocol utilized to prepare the specimen, nonrigid registration is advantageous as the image can be warped and therefore adapted to any deformation that have occurred during the preparation steps (mostly during the chemical fixation and dehydration procedures). For samples processed near to native conditions, such as those methods utilized for cryofixation, rigid registration is most appropriate since specimen deformation or structural changes is minimal.

\section{References:}

[1] Correlative Light and Electron Microscopy: Methods in Cell Biol.Vol 111. Ed. Thomas Müller-Reichert, Paul Verkade (Elsevier Inc, Pages 1-404, 2012).

[2] Correlative Light and Electron Microscopy III: Methods in Cell Biol. Vol 140. Ed. Thomas Müller-Reichert, Paul Verkade (Elsevier Inc, Pages 1-352, 2017).

[3] CS López et al. Microscopy \& Microanalysis, 24 (S1), 2018 pp. 1222-1223.

[4] CS López et al. in Methods Cell Biol. 140:149-164 (2017).

[5] Y Zhang et al. PLoS One. 12(5), 2017.

[6] D Keene et al. Microscopy and Microanalysis, 22(S3), 206-207, (2016).

[7] JK Doh et al. Proc Natl Acad Sci U S A. 2018 Dec 18;115(51):12961-12966.

[8] Microscopy was performed at the Multiscale Microscopy Core. The authors acknowledge funding from OCSSB to CSL and NIH-NCI Cancer Center Support Grant (CCSG) 2P30CA069533.
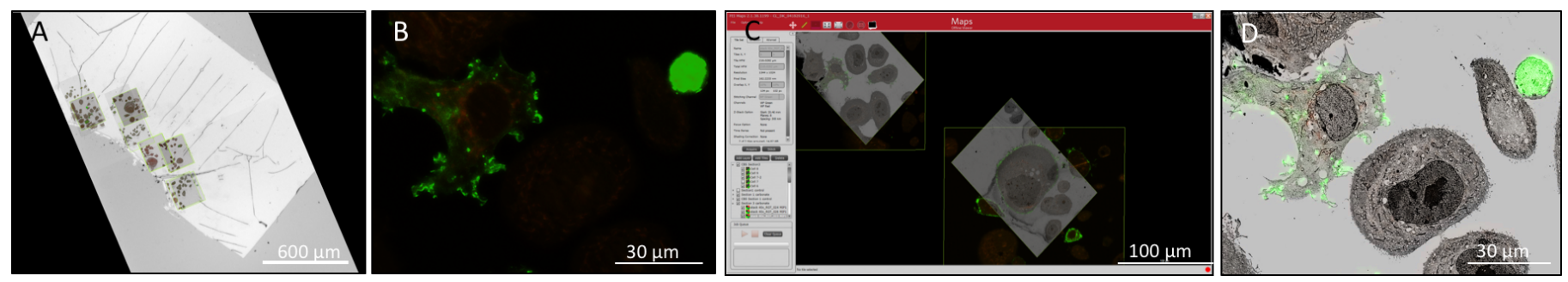

Figure 1: A) 5x bright field image showing a LRW plastic section of embedded cells mounted on ITO coverslip acquired on a CorrSight ${ }^{\mathrm{TM}}$ fluorescence microscope. Smaller images showing HER2-GFP signal. B) Cellular contour of a cell expressing HER2-GFP. C) FEI MAPS ${ }^{\text {TM }}$ software used to target the same region of interest on the LRW section. D) MAPS image overlay of fluorescence signal and backscattered electron signal acquired on a Helios Nanolab 660 DualBeam $^{\mathrm{TM}}$ Concentric BackScatter detector (CBS) at $2.5 \mathrm{kV} 0.2 \mathrm{nA}$.
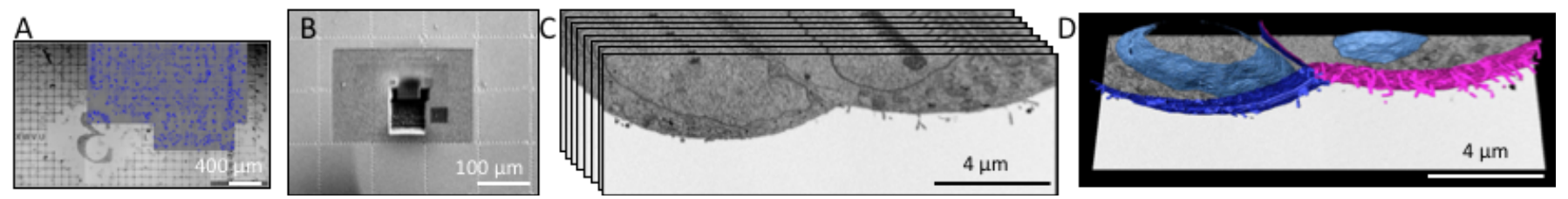

Figure 2: A) 5x bright field image showing the IBIDI grid profile and the region of interest. B) Low magnification SEM secondary electron image showing the same IBIDI grid and the selected area prepared for 3D data acquisition after MAPS registration. C) 3D volume acquisition using FEI AutoSlice and View ${ }^{\mathrm{TM}}$ software collected at $4 \mathrm{~nm}$ voxel size. D) 3D volume reconstruction. 